\title{
RESPUESTA MORFOAGRONÓMICA Y CALIDAD EN PROTEÍNA DE TRES ACCESIONES DE QUINUA (Chenopodium quinoa Willd.) EN LA SABANA NORTE DE BOGOTÁ
}

\section{MORPHOAGRONOMIC RESPONSE AND PROTEIN QUALITY OF THREE ACCESSIONS OF QUINOA (Chenopodium quinoa Willd.) IN THE NORTHERN SABANA OF BOGOTA}

\author{
Claudia Veloza Ramírez ${ }^{1}$, Gladys Romero Guerrero ${ }^{2}$, John Jairo Gómez Piedras ${ }^{3}$
}

\begin{abstract}
${ }^{1}$ Estudiante de Ingeniería Agronómica. Universidad de Ciencias Aplicadas y Ambientales U.D.C.A, calle 222 No 54-37, Bogotá, D.C., Colombia, e-mail: claudiavelozr@gmail.com; ${ }^{2}$ I.A., Ph.D. Docente Investigador, Facultad Ingeniería Agronómica U.D.C.A, e-mail: glromero@udca.edu.co; ${ }^{3}$ I.A. Docente, Facultad de Ciencias, U.D.C.A, e-mail: jgomez@udca.edu.co
\end{abstract}

Rev. U.D.C.A Act. \& Div. Cient. 19(2): 325-332, Julio-Diciembre, 2016

\section{RESUMEN}

La quinua Chenopodium quinoa Willd. es una planta dicotiledónea, considerada un pseudocereal, por las características del cultivo y formas de consumo, que ha pasado a ser de interés mundial en los últimos años, debido a su alta calidad nutritiva y de amplia adaptación ecológica. Con el objetivo de aportar al conocimiento del cultivo, se evaluaron características de rendimiento, de calidad, de variación morfológica y de desarrollo fenológico, de tres accesiones de quinua, denominadas Piartal, Nariño y Bolivia. El estudio, se llevó a cabo en la sede El Remanso de la Universidad de Ciencias Aplicadas y Ambientales U.D.C.A, ubicada en el norte de Bogotá, con coordenadas geográficas $4^{\circ} 35^{\prime}$ latitud norte y $74^{\circ} 04^{\prime}$ longitud oeste. La evaluación fue realizada durante un ciclo de producción. Los resultados indicaron diferencias en rendimiento por planta, contenido de proteína, características morfológicas de la coloración del tallo, forma de la panoja y madurez fisiológica. El rendimiento promedio varió entre 140,09 a 260,74g por planta; los mayores rendimientos se presentaron en la accesión Piartal que, a su vez, reveló el desarrollo fenológico más corto, de seis meses. Las accesiones evaluadas mostraron alta homogeneidad fenotípica al interior de cada material, lo que permite su uso en cultivos comerciales directamente y también como parental, para hibridación dentro de programas de mejoramiento genético.

Palabras clave: Fenología, componentes de rendimiento, mejoramiento, calidad nutricional.

\section{SUMMARY}

Quinoa, Chenopodium quinoa Willd. is a dicotyledoneous plant, considered a pseudocereal, according to growth characteristics and forms of consume, has become of worldwide interest in recent years due to its high nutritional quality and wide ecological adaptation. In order to contribute to the knowledge of the crop, performance characteristics, quality, morphological variation and phenological development of three accessions of quinoa, Piartal, Nariño and Bolivia were evaluated. The study was conducted under conditions of the U.D.C.A, located in the north of Bogotá, with geographical coordinates $4^{\circ} 35^{\prime}$ latitude north and $74^{\circ} 04^{\prime}$ latitude west during a production cycle. The results showed differences in plant yield, protein content, and stem color characteristics, panicle shape and physiological maturity. The yield ranged from 140.09 to $260,74 \mathrm{~g}$ per plant, the highest yield was obtained with the Piartal accession, which also showed the shortest phenological development, six months. The accessions evaluated showed high phenotypic homogeneity within materials, allowing its direct use in commercial crops, as well as parentals for hybridization in breeding programs.

Key words: Phenology, yield components, improvement, nutritional quality.

\section{INTRODUCCIÓN}

La quinua Chenopodium quinoa Willd. es una especie vegetal originaria de la región andina. Es un cultivo en expansión, dada sus características nutritivas, medicinales y de adaptación a diferentes zonas agroecológicas; se documenta, como el único vegetal que provee todos los aminoácidos 
esenciales (Gonzales et al. 2012). Contiene ácidos grasos, vitaminas y minerales, que la hace un complemento nutricional para el desarrollo y múltiples funciones del organismo; también, se le atribuyen propiedades medicinales, como cicatrizantes, desinflamatorias, analgésicas y desinfectantes (Mujica \& Jellen, 2006).

Los procesos evolutivos y la diversidad genética han logrado la domesticación de muchas especies, entre ellas, la quinua, que se adapta a distintas condiciones agroecológicas, dada a su plasticidad (Mujica \& Jellen, 2006). Se ha reportado la mayor diversidad de genotipos y progenitores silvestres, específicamente, en los alrededores del lago Titicaca, en Perú y Bolivia; de allí, se facilitó su distribución a los países andinos (Apaza et al. 2013).

La diversidad genética de la quinua, se asocia con cinco ecotipos (Jellen, 2014): Altiplano, en donde esta Perú y Bolivia; Valles_Inter-andinos, comprendido por Colombia, Ecuador y Perú; Salares, tierras de Bolivia, Chile y Argentina y Yungas, ubicado en Bolivia, zonas costeras y tierras bajas de Chile. Los valles inter- andinos están en zonas entre los 2.200 y 3.500 msnm y las plantas se caracterizan por tener un hábito de crecimiento ramificado hasta el segundo tercio, con una altura mayor a $1,5 \mathrm{~m}$; presenta abundante follaje y se desarrolla en franjas con altas precipitaciones y su ciclo vegetativo y maduración fisiológica es superior a 5 meses (Rojas et al. 2014).

Es una planta dicotiledónea, de la familia Amarantaceae, herbácea, anual o bianual; su morfología, coloración y fenología depende de las zonas agroecológicas donde se cultiva, ya que tiene una alta plasticidad para adaptarse a diferentes condiciones edáficas, sequía, heladas y salinidad (Apaza et al. 2013). La adaptabilidad, se adquirió por procesos evolutivos, que implicaron cruzamientos con Chenopodium carnosulum, por ello, su resistencia a la salinidad; con Chenopodium petiolare, para tolerar la sequía y con Chenopodium pallidicaule, su resistencia a heladas (Mujica \& Jellen, 2006). Su periodo vegetativo puede variar desde 90 hasta 240 días; se desarrolla, adecuadamente, con precipitaciones de 200 a $280 \mathrm{~mm}$ anuales; se adapta a cualquier textura de los suelos y niveles de $\mathrm{pH}$, entre 4,5 y 9,0 y en su madurez fisiológica presenta varias coloraciones, dependiendo del genotipo (Apaza et al. 2013).

La diversidad genética inter e intraespecífica, así como entre cultivares, puede ser explicada por características fenotípicas, razón, por la cual, la caracterización morfoagronómica permite suministrar información sobre la identidad de cada una de las entradas o accesiones, a través del uso de descriptores, con el fin de contar con información base, para iniciar un programa de mejoramiento genético, establecimiento y purificación de bancos de germoplasma (Ramos \& Queiroz, 1999; Becerra \& Paredes, 2000).
La quinua fue consumida por los habitantes prehispánicos de los pueblos indígenas de las tierras altas de los Andes (Colombia, Argentina y Chile), hasta la llegada del trigo a estas regiones, también por el aumento en la siembra de cebada y pastoreos. El cultivo de esta planta disminuyó, a causa de la falta de información y de conocimiento; por ejemplo, la mezcla indiscriminada de accesiones provenientes de múltiples lugares, produjo un material de baja calidad, lo que acarrea altos costos de producción, poca rentabilidad y bajos rendimientos, al no contar con variedades de quinua homogéneas, que permitan al agricultor saber el comportamiento del cultivo.

La falta de transferencia de tecnología e investigación no permite que haya mejor aprovechamiento; se presentan altas pérdidas en cosecha y post cosecha, por la falta de maquinaria, métodos para el trillado y transformación del grano. La enfermedad más importante reportada en quinua es el mildiu, causado por Peronospora farinosa chenopodii, que afecta, principalmente, el follaje y puede disminuir el rendimiento de la planta (Danielsen \& Ames, 2014).

La distribución geográfica de la quinua en América del sur comprende a los países de Colombia, Ecuador, Perú, Bolivia, Argentina y Chile, con una altitud variable, desde cero hasta los $4.000 \mathrm{msnm}$. A partir de dicho acervo genético, se ha expandido, a nivel mundial, como cultivo comercial, en algunos países de América del Norte, Europa, Asia y África (Apaza et al. 2013).

En Colombia, los indígenas, como los Chibchas y habitantes de la meseta Cundiboyacense, cultivaban y consumían la quinua, tanto para comercio (trueque) como rituales de fertilidad de sus cultivos. También, en la zona del Huila, en San Agustín, se intercambiaban productos con la sabana de Bogotá y se cree que ayudaron con la dispersión de la quinua hacia Nariño sur de Colombia y de ahí hacia Ecuador (Tapia, 2014).

En el altiplano Cundiboyacense, la actividad agrícola se basa, esencialmente, en el cultivo de la papa, con cerca de 130.000ha (ICA, 2013), un cultivo intensivo con baja rotación, presentando efectos negativos para los ecosistemas y el manejo de plagas y de enfermedades. Se hace necesaria la diversificación de la agricultura y, por consecuencia, de la oferta alimenticia. Una de las alternativas es el cultivo de la quinua, por sus características nutricionales, de adaptación y valor social.

En la actualidad, se siembra quinua en los departamentos de Nariño, Cauca, Boyacá y Cundinamarca. En Boyacá, se reportan cultivos en la provincia centro, especialmente, en municipios de Toca, Chivata, Siachoque, Soraca, en un rango de altitud variable, desde 2.760 hasta $2.810 \mathrm{msnm}$; en 
la provincia Tundama, en el municipio de Belén, con una altitud de 2.650msnm; en la provincia Sugamuxi; en los municipios de Mongui, Topaga, Tibasosa, Gameza, Sogamoso y Aquitania, varía de una altitud desde 2.538 hasta 3.031 msnm; sin embargo, no existen estadísticas actualizadas de áreas cultivadas.

Los estudios de desarrollo del cultivo, en nuestro país, son deficientes. Relacionado con la caracterización accesiones y evaluación de líneas, se reportan los trabajos de Torres et al. (2000), quienes desarrollaron una evaluación de 19 accesiones en la Sabana de Bogotá, encontrando alta variabilidad, en cuanto a rendimiento en grano, biomasa, precocidad y porte bajo. Delgado et al. (2009) evaluaron 16 líneas de quinua dulce, pertenecientes a los genotipos Piartal, Tunkahuan y Blanca de Jericó, en cuanto a sus componentes de rendimiento y estados fenológicos, en el departamento de Nariño, encontrando líneas precoces menores a 130 días, con buenos rendimientos. Cortes \& Rubiano (2007) estimaron el comportamiento agronómico de tres ecotipos de quinua, en dos municipios del centro de Cundinamarca y no encontraron diferencias en sus ciclos fenológicos; sin embargo, no se documenta procesos de selección ni mejoramiento genético de variedades, a partir de los anteriores resultados.

En general, para el país, es un cultivo de pequeña escala, con bajo nivel de tecnificación; no se cuenta con variedades seleccionadas y caracterizadas; el cultivo, se hace a partir de la mezcla de semillas, situación que afecta los rendimientos y la calidad del grano. Así, el objetivo del trabajo fue evaluar el comportamiento morfoagronómico de tres accesiones de grano blanco, asociado a bajos contenidos de saponina y, de esta forma, aportar al conocimiento del cultivo, lo que aumenta las alternativas de rotación y de la región Cundiboyacense.

\section{MATERIALES Y MÉTODOS}

La evaluación, se llevó a cabo en la sede El Remanso de la universidad de Ciencias Aplicadas y Ambientales U.D.C.A, ubicada en la sabana norte de Bogotá, a una altitud de 2.560 msnm, con coordenadas geográficas de $4^{\circ} 35^{\prime}$ latitud Norte y $74^{\circ} 04^{\prime}$ longitud Oeste. Las condiciones climáticas para el periodo de evaluación, se caracterizaron por una temperatura media de $14,8^{\circ} \mathrm{C}$ y una precipitación de $877 \mathrm{~mm}$, en el 2014 y 506,5mm, en el 2015, con una distribución bimodal.

Se analizaron tres accesiones de quinua de grano blanco, cultivadas en la Sabana de Bogotá y en el departamento de Nariño y una con origen boliviano, denominadas Piartal, Nariño y Bolivia, respectivamente. Las dos primeras, adquiridas por productores de Cundinamarca e Ipiales; la accesión Bolivia fue donada por expositores Bolivianos, evento realizado en el Jardín Botánico de Bogotá. El experimento, se sembró en un diseño completamente aleatorio, donde los tratamientos fueron las accesiones, el área total de evaluación por cada accesión, que varió de 16 a $44 \mathrm{~m}^{2}$, dependiendo de la disponibilidad de semilla, la unidad de observación fue la planta, para un total de 100 plantas valoradas, por cada accesión. La siembra, se realizó en surcos a chorrillo, con una distancia de $0,8 \mathrm{~m}$. El suelo presentó un $20 \%$ de materia orgánica, pH de 7,04 y conductividad eléctrica de 0,6ds/m, textura franco-limosa; las prácticas agronómicas correspondieron al control de arvenses y no se realizó fertilización.

Se registró información de características, según los descriptores para quinua, propuestos por Bioversity International, FAO, PROIMPA, INIAF y FIDA (2013). Se evaluaron las variables: color de la panoja en floración (CPF) y en maduración fisiológica (CPM), utilizando el círculo cromático natural color system, designado para vegetales; forma de la panoja (FDP); color de estrías (CE) y color de axilas (CA), en estado de floración. Para los estados fenológicos, se cuantificó los días a seis hojas verdaderas (SHV), días a ramificación (DR), días a panojamiento (DP), días a floración (DF), días a grano lechoso (DGL), días a grano pastoso (DGP), días a madures fisiológica (MF); cada una de las fases, se tomó desde la siembra. En cuanto a los componentes de rendimiento, se midieron las variables: número de ramas (NR), rendimiento por planta (peso del grano seco en gramos por planta) (RP), peso de 1.000 granos (WG), Índice de cosecha (IC), según Bhargava et al. (2006).

Se tasó el contenido de proteína total en grano (PT) y en residuos de trilla, conocido como perigonio (PTP) sobre base seca, para las tres accesiones. La determinación de proteína, se realizó mediante digestión de la muestra con ácido sulfúrico, destilación en ácido bórico y titulación con ácido clorhídrico de normalidad 0,1 certificado, de acuerdo a la AOAC International (2003).

Se realizó un análisis descriptivo para las diferentes variables evaluadas; luego de verificar los supuestos para análisis paramétricos, se hizo transformación mediante raíz cuadrada de las variables rendimiento por planta (RP) e índice de cosecha (IC) y se practicó análisis de varianza y prueba de comparación múltiple de Tukey; las variables número de ramas (NR) y peso de 1.000 granos (WG), así como todas las variables de fenología, no cumplieron los supuestos y se optó por comparación, mediante la prueba de Kruskal-Wallis. Finalmente, para observar en conjunto la diferenciación de las accesiones, se ejecutó un análisis de componentes principales. Los análisis se realizaron con el paquete estadístico R Core Team, 2015. 


\section{RESULTADOS Y DISCUSIÓN}

Estados fenológicos y morfología: Las accesiones evaluadas llegaron al estado de seis hojas verdaderas (SHV), alrededor de 30 DDS, sin presentar evidencia de diferencias estadísticamente significativas, en tanto que las variables días a ramificación (DR), días a panojamiento (DP), días a floración (DF), días a grano lechoso (DGL) y días a grano pastoso (DGP), presentaron diferencias para las accesiones Nariño y Bolivia, respecto de Piartal (Tabla 1). La accesión Piartal, se caracterizó por lograr su madurez fisiológica (MF), a los 180 días, mientras que las accesiones Nariño y Bolivia alcanzaron su madurez a los siete meses, después de la siembra. Evaluaciones realizadas en el departamento de Nariño, a una altura de $2.400 \mathrm{msnm}$, en selecciones de Piartal y materiales adaptados a la zona, encontraron ciclos a madurez fisiología de 160 a 178 días (Jacobsen et al. 2001). La quinua por su diversidad y evolución presenta alta adaptabilidad y plasticidad fenológica, encontrando accesiones que llegan a madurez fisiología desde los 119 a los 220 días, presentando diversidad en los ciclos productivos de cada accesión, debido a la genética de cada genotipo junto con las condiciones edafoclimáticas presentes en el desarrollo (Jacobsen et al. 2001).

La fenología de la quinua, se divide en tres fases (Figura 1): la fase vegetativa, comprendida desde los 15 DDS (días después de la siembra) hasta la ramificación; las accesiones variaron entre los 70 a 90 DDS. La fase reproductiva, comprendida entre el panojamiento y el estado fisiológico de grano pastoso, para el cual, las accesiones estuvieron entre 150 y 177 DDS y la fase de desarrollo y madurez fisiológica, caracterizada por una panoja con colores opacos, desde amarillos a cafés, la consistencia del grano es dura y no presenta cambio al ser oprimido (Gómez \& Castellanos, 2016). Se han caracterizado accesiones de quinua, que presentan madurez fisiología desde los 119 hasta los 220 días (Rojas et al. 2014), por lo que las accesiones evaluadas pueden ser catalogadas como tardías, con posible origen en los valles interandinos, debido a su rango de distribución, hábitos de crecimiento ramificado y adaptación a altas precipitaciones.

Respecto a las variables morfológicas, las tres accesiones presentaron estrías, la accesión Piartal mostraron estrías de color verde (G40Y), según la tabla de natural color system y las demás accesiones con verde (G50Y). La accesión Bolivia y Nariño muestran axilas pigmentadas de color violeta (R20B), por el contrario, la accesión Piartal, no las presenta. Las panojas de las accesiones Piartal y Bolivia, se caracterizaron por ser gloméruladas, sus ejes son insertos y cortos, lucen una forma globosa; en cuanto al color en estado de floración, varií entre violeta (R40B) y verde (G30Y), respectivamente. La accesión Nariño presentó una panoja amarantiforme distinguida por que los glomérulos están insertos directamente en el eje secundario y exhiben una forma alargada, forma de panoja, característica de Quinua real, denominación dada por el tamaño de grano grande; el color de panoja en floración es similar a Piartal. Estudios indican que en la etapa de panoja e inicio de floración es posible observar variabilidad en color de verde, purpura a rojo; sin embargo, a medida que avanza el desarrollo hacia la fase de madurez fisiología, se presenta una mayor diversidad de colores, combinando claros a oscuros, cremas, amarillos, rojos, café hasta negro (Rojas et al. 2014).

Componentes de rendimiento y calidad: Para la variable número de ramas (NR), se encontró evidencia de diferencias altamente significativas entre las accesiones Piartal y Bolivia, respecto de Nariño, siendo el promedio 19,4 número de ramas menor, para esta última (Tabla 2). El peso de 1.000 granos (WG) mostró diferencias significativas, según prueba de comparación múltiple de Tukey, en donde observa que la accesión Bolivia refleja el menor peso (Tabla 2). El rendimiento por planta (RP), así como el índice de cosecha (IC), fueron superiores para la accesión Piartal, con $260,7 \mathrm{~g} /$ planta y $41,5 \%$, en tanto que la variedad Nariño presento el

Tabla 1. Resultados de la comparación de promedios de estados fenológicos de las accesiones de quinua.

\begin{tabular}{|c|c|c|c|c|c|c|c|}
\hline \multirow{2}{*}{ Accesión } & \multicolumn{7}{|c|}{ Estados Fenológicos (DDS) } \\
\cline { 2 - 8 } & SHV & DR & DP & DF & DGL & DGP & MF \\
\hline Piartal & $30,0 \mathrm{a}^{*}$ & $45,0 \mathrm{a}$ & $70,0 \mathrm{a}$ & $100,0 \mathrm{a}$ & $120,0 \mathrm{a}$ & $150,0 \mathrm{a}$ & $180,0 \mathrm{a}$ \\
\hline Nariño & $30,8 \mathrm{a}$ & $50,6 \mathrm{~b}$ & $80,8 \mathrm{~b}$ & $115,8 \mathrm{~b}$ & $146,8 \mathrm{~b}$ & $176,8 \mathrm{~b}$ & $210,8 \mathrm{~b}$ \\
\hline Bolivia & $29,8 \mathrm{a}$ & $49,8 \mathrm{~b}$ & $79,8 \mathrm{~b}$ & $114,8 \mathrm{~b}$ & $145,8 \mathrm{~b}$ & $175,8 \mathrm{~b}$ & $209,8 \mathrm{~b}$ \\
\hline
\end{tabular}

SHV, Seis hojas verdaderas; DR, días a ramificación; DP, días a panojamiento; DF, días a floración; DGL, días a grano lechoso; DGP, días a grano pastoso; MF, días a madures fisiológica. "Promedios seguidos de la misma letra no presentan evidencia de diferencias estadísticamente significativas por la prueba de Kruskal-Wallis con una confianza del 95\%. 


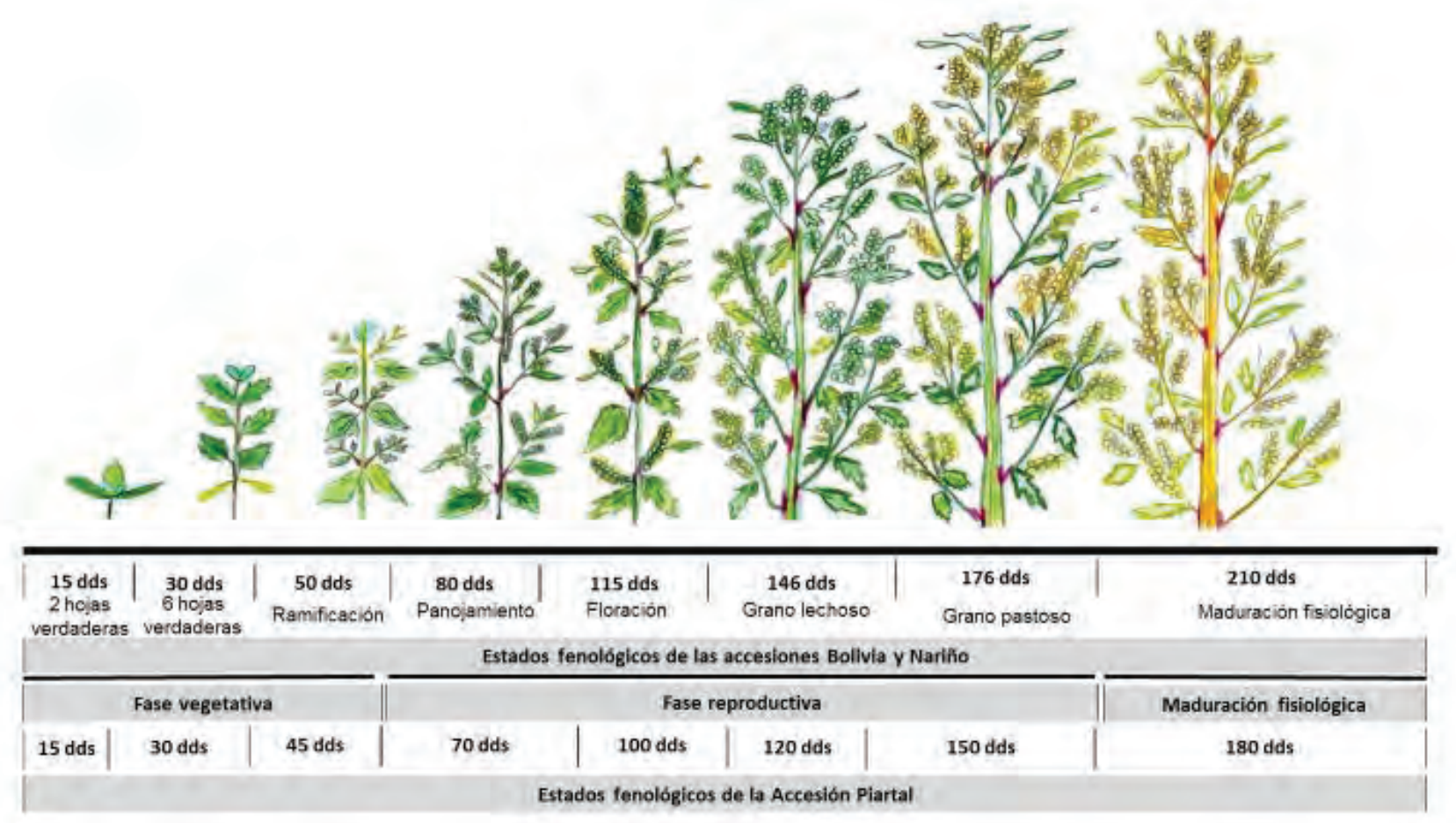

Figura 1. Estados fenológicos de tres accesiones de quinua, en condiciones en la Sabana Norte de Bogotá.

menor rendimiento, con 140,0 g/planta, mientras que no se encontró evidencia de diferencias significativas entre los índices de cosecha de Nariño y Bolivia (Tabla 2). Los registros de quinuas en Bolivia indican rendimientos promedio de $250 \mathrm{~g} \mathrm{e}$ IC de $40 \%$; las caracterizaciones practicadas para accesiones Bolivianas indican que el IC puede variar desde 6 hasta $87 \%$ (Rojas et al. 2014), por lo cual, es posible indicar que las accesiones evaluadas manifiestan potencial para la producción extensiva, ya que sin manejo de fertilización expresaron rendimientos e índices de cosecha, dentro de los promedios reportados. Los componentes de rendimiento son determi- nantes de la calidad comercial del cultivo y son criterios de selección de materiales potenciales (Zurita et al. 2014).

La cuantificación del contenido de proteína en grano indicó que el mayor porcentaje lo presenta la accesión Piartal, con $16,3 \%$, mientras Nariño el menor valor (Figura 2). Los contenidos de proteína en colecciones Bolivianas oscilan entre 10 y 18\% (Rojas et al. 2014), mientras que en quinuas, de origen peruano, se ha reportado hasta un $21 \%$ de proteína, pero asociado a granos de color oscuro (Bhargava et al. 2007), probablemente con altos contenidos de saponina.

Tabla 2. Resultados de la comparación de medias de características de rendimiento y de calidad de proteína, en tres accesiones de quinua.

\begin{tabular}{|c|c|c|c|c|}
\hline \multirow{2}{*}{ Accesión } & & \multicolumn{3}{|c|}{ Componentes Rendimiento } \\
\cline { 2 - 5 } & NR & RP $[\mathbf{g} /$ planta] & WG [g] & IC[\%] \\
\hline Piartal & $24,7 \mathrm{a}^{*}$ & $260,7 \mathrm{a}^{*}$ & $2,9 \mathrm{a}^{* *}$ & $41,5 \mathrm{a}^{*}$ \\
\hline Nariño & $19,4 \mathrm{~b}$ & $140,0 \mathrm{~b}$ & $2,9 \mathrm{a}$ & $34,2 \mathrm{~b}$ \\
\hline Bolivia & $24,6 \mathrm{a}$ & $169,1 \mathrm{c}$ & $2,8 \mathrm{~b}$ & $34.2 \mathrm{~b}$ \\
\hline
\end{tabular}

NR, número de ramas; RP, rendimiento por planta; WG, peso de 1000 granos; IC, Índice de cosecha. Promedios seguidos de la misma letra no presentan evidencia de diferencias estadísticamente significativas por la prueba de "Tukey HSD o "KruskalWallis con una confianza del $95 \%$. 
Es importante indicar que en los residuos de trilla, el perigonio, un subproducto, se presentaron altos contenidos de proteína, superior al $10 \%$, con lo que se reconoce potencial y se recomienda evaluar para ser incorporado en bloques nutricionales para animales; también puede tener potencial como sustrato en la producción de hongos comestibles y germinación de semillas, por su estructura gruesa, para dar aireación y retención de humedad.

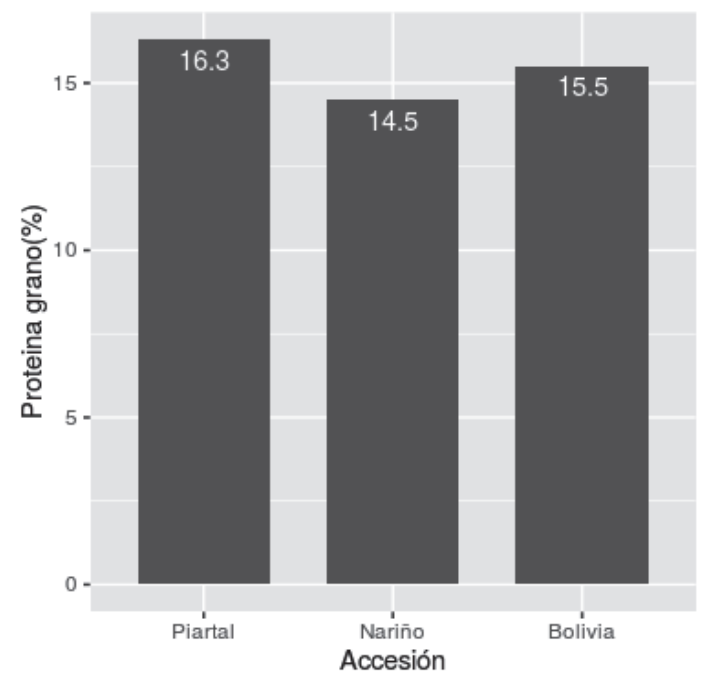

Componentes principales: El análisis de componentes principales para variables de rendimiento y características morfológicas de coloración y de forma de la panoja, indicó que son suficientes dos dimensiones (ejes) para explicar el $64,4 \%$ de la variación total, como se muestra en la figura 3. Se evidencian diferencias morfológicas y de componentes de rendimiento para las tres accesiones, lo que hace que se ubiquen distanciadas en el plano factorial (Figura 3A); a su vez, al in-

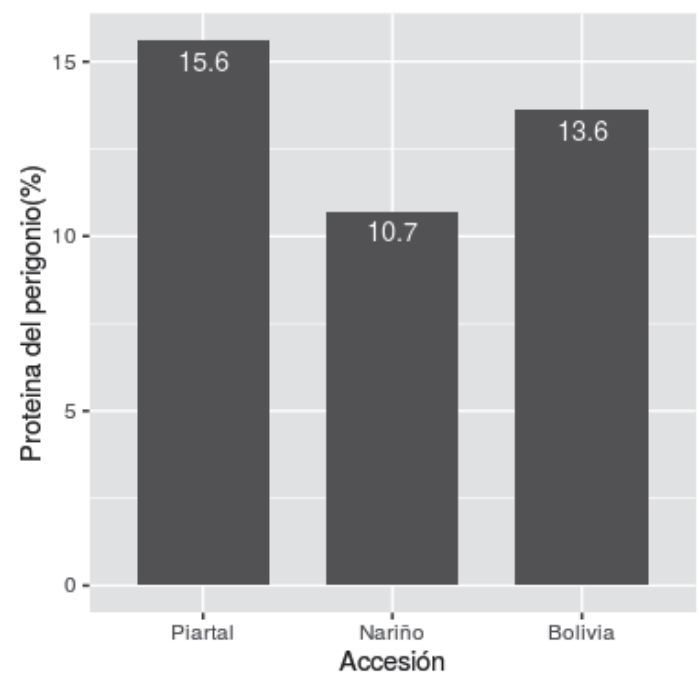

Figura 2. Comparación de los contenidos de proteína en grano y en perigonio, de las tres accesiones de quinua.

terior de cada accesión, se aprecia homogeneidad en los genotipos al ser agrupados, condición importante para realizar una caracterización y poder asociar variables morfológicas y agronómicas a los genotipos.

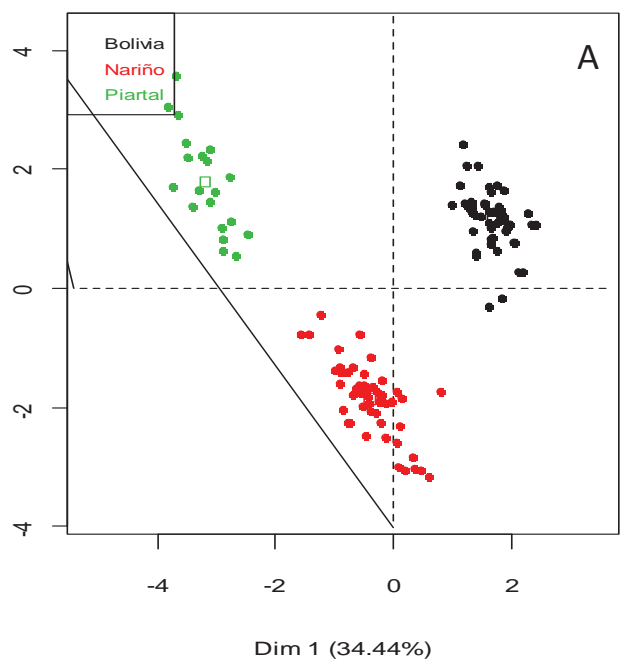

En la figura 3B, se muestra el análisis de correlaciones entre las variables que explican la variación entre accesiones. El genotipo Piartal, se destaca porque presenta los mejores componentes de rendimiento (RP, IC) y se diferencia de las

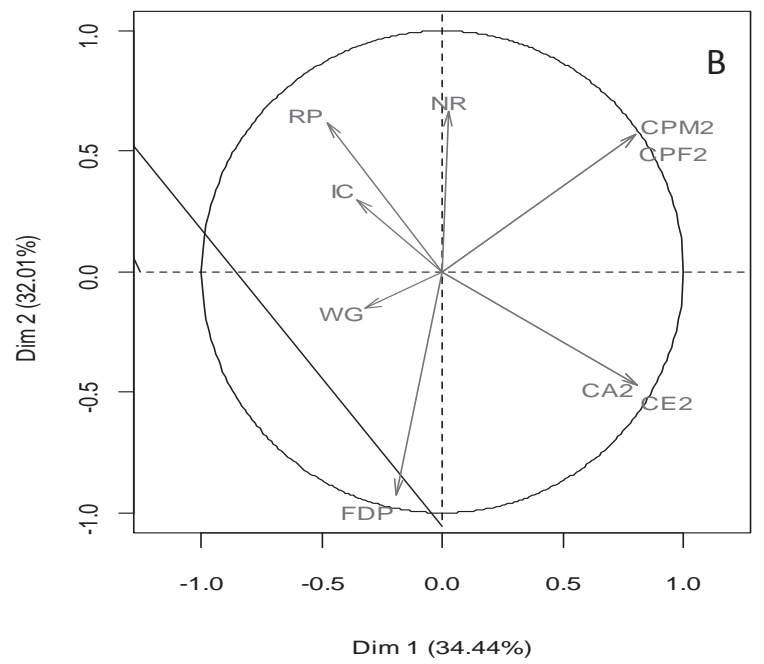

Figura 3. A) Plano factorial de análisis de componentes principales, para medidas, en tres accesiones de Quinua y B) Círculo de correlaciones para variables, medidas en tres accesiones de quinua. 
otras dos accesiones. El peso de 1.000 granos (WG) no está bien representada en el plano, debido a su corto vector y ubicación negativa; como se evidenció en los resultados, no presentó diferencias entre los materiales y no se relacionó con rendimiento, resultados similares obtenidos por Bertero et al. (2004) y Delgado et al. (2009). El número de ramas (NR) no se correlacionó con componentes de rendimiento, aunque asoció a las accesiones Piartal y Bolivia, en la dimensión 1. Las características morfológicas (CPF, CPM, CA, CE) muestran diferencias para las tres accesiones; sin embargo, no se asocian con componentes de rendimiento. Los materiales estudiados presentaron características de rendimiento y de calidad destacados, comparado con genotipos bolivianos, de amplia variabilidad.

La demanda de la quinua ha aumentado en el mundo, en los últimos años, siendo insuficiente la exportación de los países de América del Sur, a los países de América del Norte y la Unión Europea (Jacobsen et al. 2001). Lo que requiere un incremento en el área, la tecnificación y desarrollo de los cultivos, a su vez, la introducción de la quinua a otros países diferentes a la región Andina (Bazile, 2014), como lo muestran Italia y Grecia, donde se tienen resultados de rendimiento de 2 y 3t/ha (Jacobsen, 2014), manifestando la amplia adaptación de la planta a diferentes condiciones de cultivo.

Agradecimientos: Los autores expresan los agradecimientos a la Doctora Aurora Cuesta, del laboratorio de nutrición animal de la U.D.C.A, por su apoyo con la cuantificación de proteína, a la Universidad de Ciencias Aplicadas y Ambientales U.D.C.A, por la financiación y al estudiante Jean Pierre Ronderos, por su ayuda en la toma de los datos en campo. Conflicto de intereses: El manuscrito fue preparado y revisado con la participación de todos los autores, quienes manifiestan que no existe conflicto de intereses que ponga en riesgo la validez de los resultados o la publicación de este artículo.

\section{BIBLIOGRAFÍA}

1. AOAC International. 2003. Official Methods of Analysis of AOAC International $17^{\text {th }}$ edition. $2^{\text {nd }}$ Revision. Gaithersburg, USA. Association of Analytical Communities.

2. APAZA, V.; CACERES, G.; ESTRADA, R.; PINEDO, R. 2013. Catálogo de variedades comerciales de quinua en el Perú: Instituto Nacional de Innovación Agraria (INIA). p.15-82.

3. BAZILE, D. 2014. Dinámica de expansión mundial del cultivo de la quinua respecto a su alta biodiversidad. Chile- Francia: CIRAD-FAO. p.1-27.
4. BECERRA, V.; PAREDES, M. 2000. Uso de marcadores bioquímicos y moleculares en estudios de diversidad genética. Agricultura Técnica. 60(3):270-281.

5. BERTERO, H.D.; DE LA VEGA, A.J.; CORREA, G.; JACOBSEN, S.E.; MUJICA, A. 2004. Genotype and genotype-by environment interaction effects for grain yield and grain size of quinoa (Chenopodium quinoa Willd.) as revealed by pattern analysis of international multi-environment trials. Field Crops Res. 89:299318.

6. BHARGAVA, A.; SHUKLA, S.; OHRI, D. 2007. Genetic variability and interrelationship among various morphological and quality traits in quinoa (Chenopodium Willd.). Field Crops Res. 101:104-116.

7. BHARGAVA, A.; SHUKLA, S.; OHRI, D. 2006. Karyotipoc studies on some cultivated and wild species of Chenopodium (Chenopodiaceae). Genet. Res. Crop Evol. 53:1309-1320.

8. BIOVERSITY INTERNATIONAL; FAO; PROINPA; INIAF; FIDA. 2013. Descriptores para quinua Chenopodium quinoa Willd. y sus parientes silvestres. RomaItalia-La Paz-Bolivia-Chile: Bioversity International. p.30-40.

9. CORTES, A.; RUBIANO, A. 2007. Caracterización de tres ecotipos de Quinua Chenopodium quinoa Willd mediante técnicas agroecológicas, en dos zonas agroclimatologicamente diferentes del Departamento de Cundinamarca. Inventum. 4(2):1-10.

10. DANIELSEN, D.; AMES, T. 2014. Mildiu Peronospora farinosa de la quinua Chenopodium quinoa Willd. en la zona andina, Manual técnico para el estudio de la enfermedad y el patógeno. Perú: Centro Internacional de la papa (CIP) Royal Danish ministry of foreign affairs the royal veterinary and agricultural university. p.1-11.

11. DELGADO P., A.I.; PALACIOS C., J.H.; BETANCOURT G., C. 2009. Evaluación de 16 genotipos de quinua dulce (Chenopodium quinoa Willd.) en el municipio de Iles, Nariño (Colombia). Agronomía Colombiana. 27(2):159-167.

12. GÓMEZ, L.; CASTELLANOS, E. 2016. Guía de cultivo de la quinua. Lima-Peru: Universidad Nacional Agraria La Molina Programa de Investigación y Proyección Social de Cereales y Granos Nativos Facultad de Agronomía. p.17-24. 
13. GONZALES, J.; KONISHI, Y.; VALOY, M.; PADRO, F. 2012. Interrelationships among seed yield, total protein and amino acid composition of ten quinoa Chenopodium quinoa Willd. cultivars from two different agroecological regions. J. Science Food Agriculture. 92(6):1222-1229.

14. ICA, 2013. ICA advierte sobre el riesgo sanitario en los cultivos de papa, cebolla y ajo que no se recojan a tiempo. Minagricultura, disponible desde Internet en: http://www.ica.gov.co/Noticias/Agricola/2013/ICAadvierte-sobre-el-riesgo-sanitario-en-los-cult.aspx (con acceso: 18/10/15).

15. JACOBSEN, S.E.; QUISPE, H.; MUJICA, A. 2001. Quinoa: an alternative crop for saline soils in the Andes. Scientist and Farmer, CIP Program Report 19992000. p.403-408.

16. JACOBSEN, S. 2014. Adaptación y posibilidades para la quinua en las latitudes septentrionales de Europa. Capítulo 6.11. In: Bazile, D.; Bertero, D.; Nieto, C. (eds). Estado del arte de la quinua en el mundo en 2013. FAO (Santiago de Chile) y CIRAD, (Montpellier, Francia). p.520-533.

17. JELLEN, E. 2014. Botanica, Filogenia y Evolución. En: Estado del arte de la quinua en el mundo en 2013. Chile-Francia: FAO- CIRAD. p.12-13.

18. MUJICA, A.; JELLEN, S. 2006. La quinua Chenopodium sp y sus parientes silvestres. La Paz: Universidad Mayor de San Andres. p.1-22.

19. R Core Team. 2015. R: A language and environment for statistical computing. R Foundation for Statistical Computing, Vienna, Austria. URL https://www.R-project.org/.
20. RAMOS, S.; QUEIROZ, M. 1999. Caracterizaçao morfológica: experiencia do BAG de cucurbitáceas da EMBRAPA Semiárido, com accesos de abóbora e moranga. Horticultura Brasileira. 7:9-12.

21. ROJAS, W.; PINTO, M.; ALANOCA, C.; GÓMEZ, L.; LOBOS, P.; ALERCIA, A.; DIULGHEROFF, S.; PADULOSI, S.; BAZILE, D. 2014. Estado de la conservación es situ de los recursos genéticos de la quinua. Capítulo 1.5. In: Bazile, D.; Bertero, D.; Nieto, C. (eds). Estado del arte de la quinua en el mundo en 2013. FAO (Santiago de Chile) y CIRAD, (Montpellier, Francia). p.65-94.

22. TAPIA, M. 2014. El largo camino de la Quinoa: ćquiénes escribieron su historia? In: Bazile, D.; Bertero, D.; Nieto, C. (eds). Estado del arte de la quinua en el mundo en 2013. FAO (Santiago de Chile) y CIRAD, (Montpellier, Francia). p. 3-10.

23. TORRES, J.; VARGAS, H.; CORREDOR, G.; REYES, L. 2000. Caracterizacion morfoagronomica de diecinueve cultivares de quinua Chenopodium quinoa Willd. en la sabana de Bogotá. Agronomía Colombiana. 17:60-68.

24. ZURITA, A.; JACOBSEN, S.; RAZZAGHI, F.; ÁLVAREZ, R.; RUIZ, K., MORALES, A.; SILVA, H. 2014. Respuestas a la sequia y adaptación de la quinua. Capitulo 2.4. In: Bazile, D.; Bertero, D.; Nieto, C. (eds). Estado del arte de la quinua en el mundo en 2013. FAO (Santiago de Chile) y CIRAD, (Montpellier, Francia). p.185-202.

Recibido: Octubre 5 de 2016

Aceptado: Noviembre 30 de 2016

Cómo citar:

Veloza Ramírez, C.; Romero Guerrero, G.; Gómez Piedras, J.J. 2016. Respuesta morfoagronómica y calidad en proteína de tres accesiones de quinua (Chenopodium quinoa Willd.) en la Sabana norte de Bogotá. Rev. U.D.C.A Act. \& Div. Cient. 19(2): 325-332. 